\title{
Application of Sequential Quadratic Programming Method to Temperature Distribution Control in Reactor Furnace
}

\author{
Kazuhito ISHIMARU, Masami KONISHI, ${ }^{1)}$ Jun IMAI ${ }^{1)}$ and Tatsushi NISHI ${ }^{11}$ \\ Graduate School of Natural Science and Technology, Okayama University, 3-1-1 Tsushimanaka, Okayama $700-8530$ Japan. \\ 1) Department of Electrical and Electronic Engineering, Okayama University, 3-1-1 Tsushimanaka, Okayama $700-8530$ Japan.
}

(Received on September. 30, 2004; accepted in final form on December 22, 2004)

\begin{abstract}
Recently, various studies have been undertaken to examine the inner furnace phenomena of a blast furnace. These studies are limited, however, to the evaluation of furnace performance while a designed method of furnace control remains unfulfilled. This paper proposes a method of estimating temperature distribution in a reactor furnace using boundary data in order to control inner target furnace temperature distribution. Initially, a simplified furnace simulation program for the calculation of inner furnace gas flow, pressure and temperature distribution is developed. Following that, the simulator is used to estimate and control inner furnace temperature distribution. In the estimation, boundary data such as temperature and pressure, measured near a furnace wall, are used in the furnace simulation. Then, for the control of inner furnace temperature distribution, necessary values for gas blowing at the bottom of the furnace and the burden supply at the top of the furnace are used. Both for the estimation and the control of the inner furnace temperature distribution, a sequential quadratic programming method, a method of the iterative optimization, is applied. In our method, an estimation of inner furnace temperature distribution is done as preparation for the determination of furnace control variables. Through numerical experiments, the validity of our method is demonstrated, showing that the temperature distribution in a furnace can be regulated to the desired one after iterative control operations.
\end{abstract}

KEY WORDS: sequential quadratic programming method; optimization; reactor furnace; temperature distribution.

\section{Introduction}

Reactor furnaces such as a blast furnace have been playing vital roles in metal industries. Based on the improvements of furnace facilities, the size of a furnace has enlarged remarkably. As is well known, internal phenomena of reactor furnaces are complex, ${ }^{1-4)}$ and the operation of a furnace still depends on the experience and intuition of skilled operators.

Since various mutable factors, such as gas flow, chemical reactions, burn-through and anastomoses of iron ores and movement of a filling in a furnace, are intricately entangled, the inner reactions of a reactor furnace remain a mystery. Meanwhile, stable, low cost furnace operation is required in the industry. Moreover, in the future, automated furnace operation will be expected to cope with a decreasing number of skilled operators and the difficulty in finding their successors.

In this research, we pursued a design method for temperature distribution control systems for reactor furnaces. As described above, due to the complexity of inner furnace phenomena such as chemical reactions, high pressure and high temperature, variables only near the furnace wall can be measured. Therefore, we tried to estimate the inner furnace temperature distribution using measured data taken from sensors equipped near the furnace wall. In the estimation, the blowing condition is regulated using the sequential quadratic programming method (SQP method) so that the measured values match the calculated values of corresponding points in the furnace. In the control of inner furnace temperature distribution, calculated distribution is compared with its desired one. In order to minimize the difference between them, blowing condition and burden supply condition are changed. Again, the SQP method is employed to carry out the control.

For these purposes, a simulator for inner furnace gas flow and temperature distribution was developed, which uses boundary conditions as control variables. This simulator is simplified from previous studies ${ }^{5-7)}$ to attain swift computation preserving the principal reaction and heat transfer phenomena in a reactor furnace.

This simplified simulator is combined with the SQP method. That is, the temperature distribution in a reactor furnace is estimated by SQP method using only measured boundary values of a furnace. Here, the measured boundary data includes temperature, pressure and gas flow near a furnace wall. Temperature distribution in a reactor furnace is controlled by regulating blowing conditions in multiple tuyeres at the furnace bottom and supplying materials at the furnace top.

\section{Control Method of Temperature Distribution}

In the following, the control method of temperature dis- 


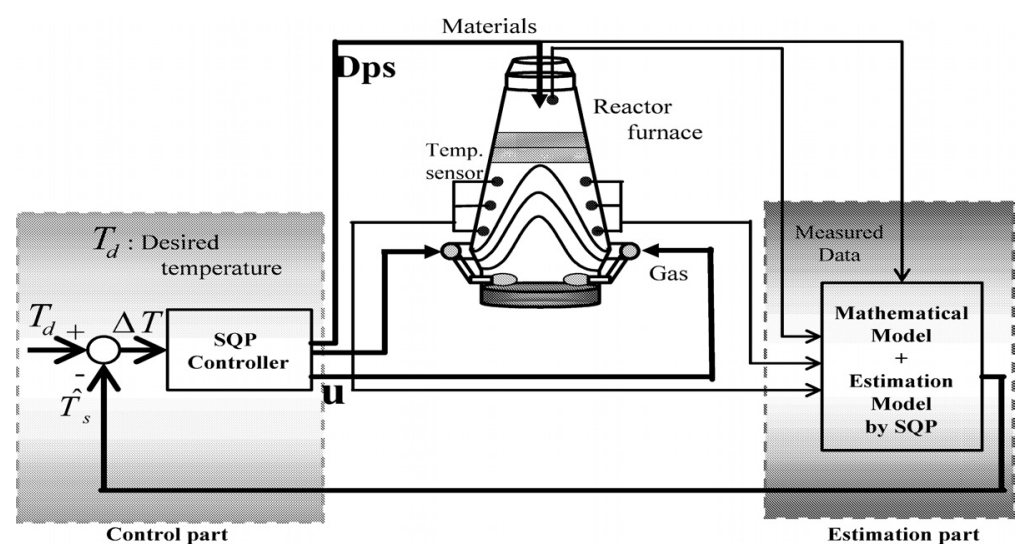

Fig. 1. Control method of temperature distribution in a reactor furnace.

tribution in a reactor furnace is described. This method is shown in Fig. 1. As shown in the figure, there are two parts, the estimation of temperature distribution and the control of it.

First, estimation part is processed. To know the temperature distribution in a reactor furnace, the mathematical model is applied using total gas flow, material charge condition and boundary conditions as its input. The mathematical model is formed by reference. ${ }^{8)}$ The temperatures calculated by the mathematical model are compared with the measured temperatures, gas volumes from multiple tuyeres are determined in order to reduce the error between these two values. Thus, the gas volumes from multiple tuyeres are calculated. The SQP method is used to estimate temperature distribution. Descriptions of the mathematical model are written in an appendix.

Next, control part is processed. Operations of a furnace in order to conform estimated temperature distribution of a reactor furnace to the desired temperature distribution must be determined. Here, temperature distribution in a reactor furnace is operated by gas flow from multiple tuyeres at a furnace bottom and supplying material from the top of a furnace. The SQP method is also used to control temperature distribution. By SQP method, each objective function for the estimation and the control is minimized. These objective functions are designed differently for the estimatetion and the control of inner furnace temperature.

\subsection{Estimation of Inner Furnace Temperature Distri- bution}

Since the central part of a reactor furnace is at high temperature and pressure, it is difficult to measure the temperature of central part. Actually, only the temperatures of outer side in a reactor furnace can be measured. In this research, the inner temperature distribution of a reactor furnace is estimated by using only measured data. ${ }^{9)}$ In other words, calculated temperature distribution in a reactor furnace will conform to the measured temperature distribution when values of calculated temperature at the same positions conform to the measured data. The SQP method is applied as the way for conforming data.

Here, positions of the instrumentation are shown in Fig. 2. Instrumentations are set up along the wall of reactor furnace. Thermometers and pressure meters are set up at the inner side wall of a reactor furnace, and gas flow meters are

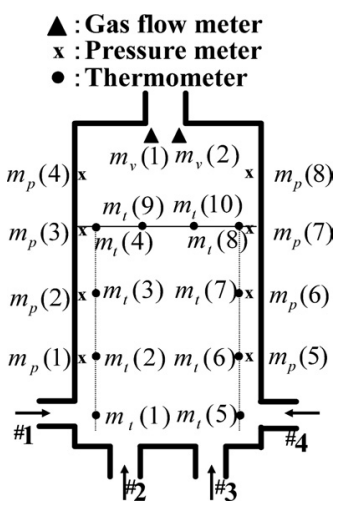

Fig. 2. Instrumentations.

set up at the top of a reactor furnace as shown in the figure.

Here, this estimation problem is formulated in the following. Decision variables are gas volumes blowing from inlet zones \#1 to \#4. When these values are decided, temperature distribution in reactor furnace can be calculated using a simulator. In the estimation, the objective function is defined by Eq. (1), that is the weighted sum of the differences in estimated values of temperature, pressure and gas flow from its measured values. The value of $f$ in Eq. (1) is to be minimized. In this problem, constraints are the upper and lower limit of gas volume and the equation for total quantity of gas and are written in Eqs. (2) to (4).

$$
f=\alpha_{\mathrm{t}} \sum_{m_{\mathrm{t}}}\left(T_{\mathrm{s}}-\hat{T}_{\mathrm{s}}\right)^{2}+\alpha_{\mathrm{p}} \sum_{m_{\mathrm{p}}}(P-\hat{P})^{2}+\alpha_{\mathrm{v}} \sum_{m_{\mathrm{v}}}(\mathbf{V}-\hat{\mathbf{V}})^{2}
$$

subject to

$$
\begin{aligned}
& c_{1 h}(u)=u_{\max }-u_{h} \geq 0 . \\
& c_{2 h}(u)=u_{h}-u_{\min } \geq 0 \\
& c_{3}(u)=\sum_{h=1}^{4} u_{h}-C=0, \quad(C: \text { constant })
\end{aligned}
$$

where, $\hat{T}_{\mathrm{s}}, \hat{P}$ and $\hat{\mathbf{V}}$ are the estimated value of solid temperatures, pressures and gas flows, respectively. $u, u_{\min }$ and $u_{\max }$ mean gas flow, lower limit of gas flow and upper limit of gas flow, respectively. And, $T_{\mathrm{s}}, P$ and $\mathbf{V}$ are the measured values of solid temperature, furnace pressure and gas flow volume, respectively. $\alpha_{\mathrm{t}}, \alpha_{\mathrm{p}}$ and $\alpha_{\mathrm{v}}$ are weighting factors. $m_{\mathrm{t}}, m_{\mathrm{p}}$ and $m_{\mathrm{v}}$ are number of instrumentation for tempera- 
ture, pressure and gas flow volume, respectively. Parameter $h$ shows number of inlet zone.

\subsection{Determination of Control Variables}

Temperature distribution in reactor furnace can be controlled by gas volume of tuyeres at the furnace bottom and supplying materials from the furnace top. ${ }^{10)}$ In this paper, a temperature control system consists of the furnace model and the SQP method. To begin with, the estimated temperature distribution in a reactor furnace at a certain time is compared with the desired temperature distribution. Then, gas volumes and size of supplying materials in the next interval is decided to minimize the temperature differences. To know the differences, the furnace simulation by applying the given gas volumes is executed. Iterating the procedure, the temperature distribution at the next interval is calculated. Operating conditions of a reactor furnace is determined by these iterative calculations.

Here, the control problem is formulated in the following. Decision variables are gas volumes in inlet zones \#1 to \#4 at the bottom of a furnace and supplying materials size from material entrance slots \#1 and \#2 at the top of a furnace at each time interval. The objective function for the temperature distribution control is defined by Eq. (5), that is the sum of the squares of temperature's differences, and the constraint of gas volume variations. Constraints are the upper and lower limit of gas volumes, the equation for total volume of gases and the upper and lower limit of supplying materials size. These constraints are written in Eqs. (6) to (10). Here, $l$ in Eqs. (5) to (10) means a sampling time of control. The control interval is set at $20 \mathrm{~h}$ which is 100 times of a time step in a furnace simulator.

$$
\begin{aligned}
f^{(l)}= & w_{1} \sum_{i=1}^{21} \sum_{j=1}^{12}\left(T_{\mathrm{d}}(i, j)-\hat{T}_{\mathrm{s}}^{(l)}(i, j)\right)^{2} \\
& +w_{2} \max \left\{\left|u_{h}^{(l)}-u_{h}^{(l-1)}\right|-a, 0\right\}
\end{aligned}
$$

subject to

$$
\begin{array}{r}
\mathrm{c}_{1 h}^{(l)}(u)=u_{\max }-u_{h}^{(l)} \geq 0 \ldots \ldots \ldots \ldots \\
\mathrm{c}_{2 h}^{(l)}(u)=u_{h}^{(l)}-u_{\min } \geq 0,(h=1,2,3,4) \\
c_{3}^{(l)}(u)=\sum_{h=1}^{4} u_{h}^{(l)}-C=0 \ldots \ldots \ldots \\
c_{4 \eta}^{(l)}(u)=D s_{\max }-D s_{\eta}^{(l)} \geq 0 \ldots \ldots \ldots . . . \\
c_{5 \eta}^{(l)}(u)=D s_{\eta}^{(l)}-D s_{\min } \geq 0,(\eta=1,2) .
\end{array}
$$

$T_{\mathrm{d}}$ : Desired temperature distribution, $w_{1}, w_{2}$ : Weighting factor, $a$ : Constant, $D s$ : Supplying materials size, $D s_{\max }$ : Upper limit of materials size, $D s_{\text {min }}$ : Lower limit of materials size, $\eta$ : Materials entrance slot, $l$ : Time interval Here, values of coefficients $w_{1}, w_{2}$ and a are set at $10^{-4}, 100$ and 1 which are empirically determined to equalize dimensions of each factor in Eq. (5).

\section{Optimization Algorithm by SQP Method}

In this research, the SQP method is used for the estimation and the control of temperature distribution. The SQP method is one of the effective methods for nonlinear programming problems. In the following, an optimization algorithm using the SQP method is described.

\subsection{About SQP Method}

Here, computing procedures of the SQP method is described.

1. The initial solution $x^{(0)}$, Hessian approximation $B^{(0)}$ and the penalty parameter $r>0$ are are set up. And $k=0$.

2. A quadratic programming problem is defined by the objective function and constrained conditions. When it is solved, $d^{(k)}=x^{(k+1)}-x^{(k)}$ and Lagrange multiplier $u^{(k+1)}$ are determined.

$$
\begin{array}{r}
\nabla f\left(x^{(k)}\right)^{\mathrm{T}} d+\frac{1}{2} d^{\mathrm{T}} B^{(k)} d \rightarrow \min . \\
c\left(x^{(k)}\right)+\nabla c\left(x^{(k)}\right)^{\mathrm{T}} d \geq 0 \ldots \ldots \ldots \ldots
\end{array}
$$

3. The penalty parameter is updated. Here, $\sigma>0$.

$$
r=\left\{\begin{array}{l}
\max \left\{\left|u_{i}^{(k+1)}\right|: i=1,2, \cdots, m\right\}+\sigma, \\
\quad \text { if } r<\max \left\{\left|u_{i}^{(k)}\right|: i=1,2, \cdots, m\right\} \ldots \ldots \\
r, \quad \text { else }
\end{array}\right.
$$

4. A linear search is done, step width $t^{(k)}>0$ is searched and $x^{(k+1)}=x^{(k)}+t^{(k)} d^{(k)}$.

5. Hessian approximation $B$ is updated by BFGS algorithm.

$$
\begin{aligned}
& B^{(k+1)}=B^{(k)}+\frac{\tilde{y}^{(k)}\left(\tilde{y}^{(k)}\right)^{\mathrm{T}}}{\left(\tilde{y}^{(k)}\right)^{\mathrm{T}} s^{(k)}}-\frac{B^{(k)} s^{(k)}\left(s^{(k)}\right)^{\mathrm{T}} B^{(k)}}{\left(s^{(k)}\right)^{\mathrm{T}} B^{(k)} s^{(k)}} \\
& s^{(k)}=x^{(k+1)}-x^{(k)} \\
& y^{(k)}=\nabla^{(k+1)}-\nabla L^{(k)} \\
& \theta= \begin{cases}1, \quad \text { if }\left(s^{(k)}\right)^{\mathrm{T}} y^{(k)} \geq 0.2\left(s^{(k)}\right)^{\mathrm{T}} B^{(k)} s^{(k)} \\
\frac{0.8\left(s^{(k)}\right)^{\mathrm{T}} B^{(k)} s^{(k)}}{\left(s^{(k)}\right)^{\mathrm{T}} B^{(k)} s^{(k)}-\left(s^{(k)}\right)^{\mathrm{T}} y^{(k)}}, & \text { else }\end{cases} \\
& \tilde{y}^{(k)}=\theta y^{k}+(1-\theta) B^{(k)} s^{(k)} \text {. }
\end{aligned}
$$

Here, $\nabla L=\nabla f-\sum_{i=1}^{M} u_{i} \nabla c$.

6. When convergence conditions is fulfilled, this calculation is finished. Otherwise $k=k+1$ and it returns to 2 .

\subsection{Estimation of Temperature Distribution by SQP Method}

In actual blast furnaces, it is impossible to measure temperatures in a reactor furnace except in the outer side of them. Because of the above situation, we constructed the estimation system using limited number of instrumentations. The method of the estimation system is shown in Fig. 3. The estimation is constructed by the measured data in limited instrumentations.

At the beginning, the initial solution and several distributions are set up as the initial conditions. Gas volumes of each tuyere is decided by the SQP method as the result of the model conforms to measured data at the same position. When sought gas volumes is applied to the model of a reac- 


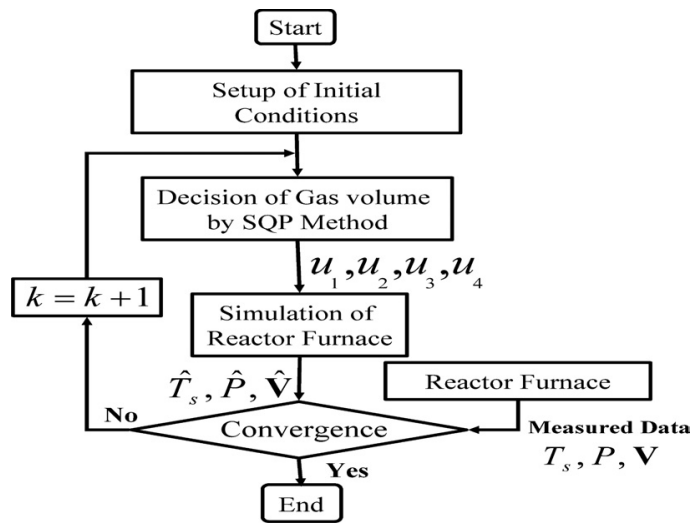

Fig. 3. Flow chart of estimation.

tor furnace, inner furnace temperature distribution is estimated. Then, the estimated value is compared with measured data. When the value of the objective function becomes to minimum, the iteration is finished. Otherwise, this procedure is iterated.

\subsection{Decision of Operation Conditions by SQP Method}

The SQP method is used to decide gas volumes. Here, the dynamic optimization algorithm to decide gas volumes by the SQP method is shown in Fig. 4.

To begin with, initial values of gas volume, a gas flow, each temperature distributions and a material charge condition are set. Then, gas volumes and supplying materials size for the next interval is decided by the SQP method. Here, the time interval is defined to be plural time steps of simulator. Decided gas volumes and supplying materials size are applied to a reactor furnace model. This results in the change of the temperature distribution in a reactor furnace and is compared with the desired temperature distribution. When the difference between calculated the temperature distribution and the desired one becomes smaller than ten degrees centigrade, the calculation is finished. Otherwise, this procedure is iterated. Here, the time step in a furnace simulator is set at $12 \mathrm{~min}$ and the sampling time is set as 100 times of the time step in a furnace simulation. Thus, the time of control interval is set at $20 \mathrm{~h}$ in our paper.

\section{Simulation of Temperature Distribution in Reactor Furnace}

\subsection{Two-dimensional Model}

This paper uses Macro-Model that calculates two-dimensional macro gas flow and temperature distribution using control factors, such as gas flow and materials size as the boundary conditions of the calculation. Nodes assignment for the two-dimensional model is shown in Fig. 5.

$x$ axis of the model is the furnace width and $y$ axis is the furnace height respectively. In a blast furnace, the furnace height is about twice of the diameter. So, the number of nodes in $x$ direction is set 12 and that in $y$ direction is set 21 as shown in Fig. 5. Here, 1 node is equivalent to $2 \mathrm{~m}$. There are 4 tuyeres in the bottom of this model and a high-temperature gas is discharged from it. Besides, a material supplying zone consist of 15 nodes as shown in Fig. 6. As shown here, materials are supplied around a reactor furnace wall.

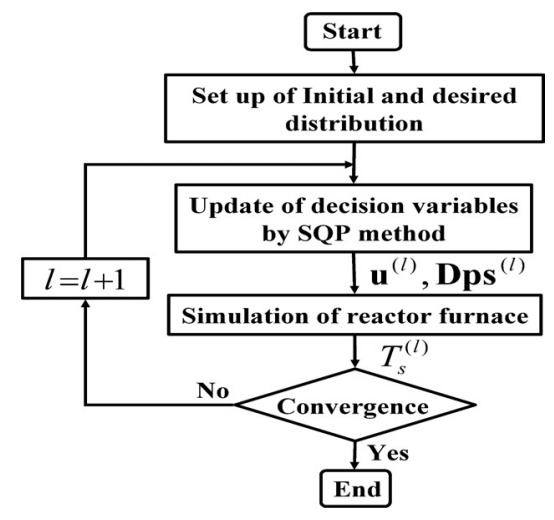

Fig. 4. Flow chart of control.

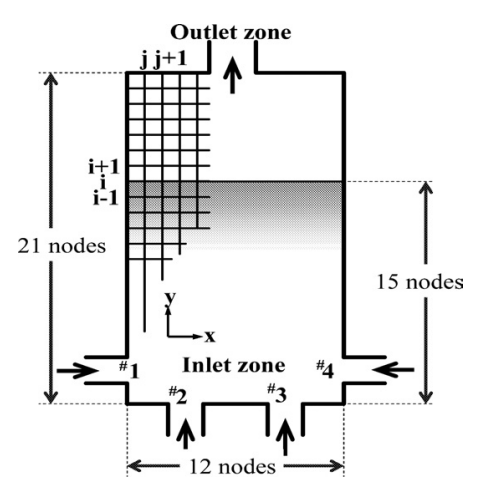

Fig. 5. Two-dimensional model.

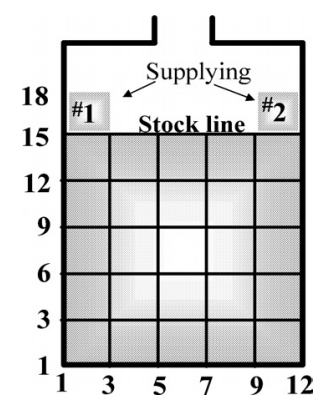

Fig. 6. Positions of supplying materials.

\subsection{Solution Procedure for Simulation}

The temperature distribution in a reactor furnace is calculated by solving the gas flow equation, the gas temperature equation and the solid temperature equation. In this paper, finite-difference approximation method is used to calculate the gas flow and the temperature distribution. The flow chart of the calculation is shown in Fig. 7.

When input values such as gas volumes of each tuyeres and supplying materials size are given, the temperature distribution in a reactor furnace is calculated. These calculations are continued until the convergence condition is fulfilled. The convergence condition is described in Eq. (19). Here, $n$ is the number of time step in the furnace simulator and set $12 \mathrm{~min}$. $T$ is the maximum value of time step.

$$
\max _{i, j}\left|T_{s}^{n+1}(i, j)-T_{s}^{n}(i, j)\right|<\sigma \quad \text { and } \quad n \geq \tau \ldots
$$

Here, $\sigma$ and $\tau$ are set at 0.5 and 100 respectively. 


\subsection{Numerical Simulation of Gas Flow and Tempera- ture}

The temperature distribution in a reactor furnace is calculated by the method stated above. Gas volumes of all tuyeres is set at 2 units and a gas temperature is set at 1200 degrees centigrade and supplying materials size is $0.045 \mathrm{~m}$ in diameter.

In the following, simulation results are shown. In Fig. 8(a), the gas volume distribution is shown and in Fig. 8(b), the solid temperature distribution is shown.

The gas flows from tuyeres at the bottom to a discharge equipment at the furnace top. The solid temperature is maximum at the center of a reactor furnace, and is gradually decreased toward the furnace wall.

\section{Numerical Experiment of SQP Application}

In Fig. 9, the flow chart of the estimation and the control is shown. In the estimation, the furnace model is used in the loop of a SQP method. Until the temperature distribution in

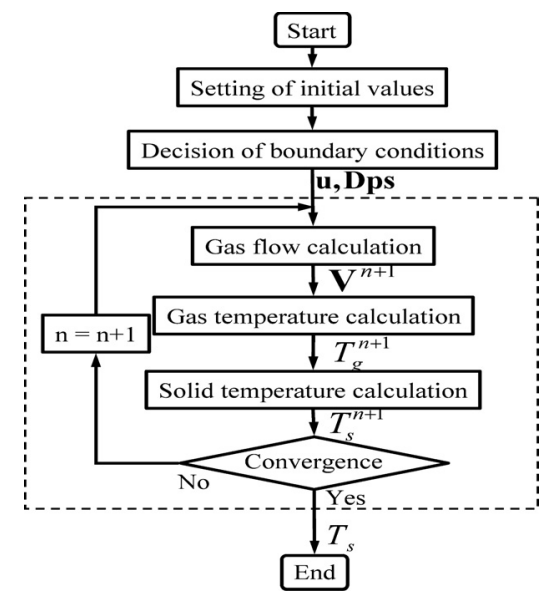

Fig. 7. Flow chart of simulation.

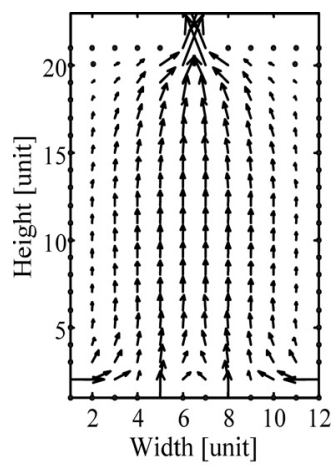

(a) Gas flow distribution

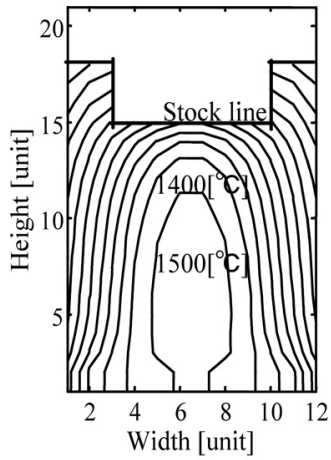

(b) Solid temperature distribution
Fig. 8. Results of furnace simulation. a reactor furnace becomes a steady state, calculation by the furnace model are continued. In the control, the furnace model is used in the loop of a SQP optimization. Calculations for one sampling time of control are carried out by the farnace model. Using the results, the temperature distribution is calculated and saved for the next control interval.

\subsection{Estimation of Temperature Distribution}

The estimation of temperature distribution in a reactor furnace is calculated by the method shown in Fig. 3 of the Sec. 3.2. Measured data of actual furnace the numerical experiments are shown in Table 1. These data are measured at nodes shown in Fig. 2. Temperatures, pressures and gas flows are measured at ten, eight and two points around a furnace. Here, the gas flow is given by vector value. Using this data, the temperature distribution in a reactor furnace is estimated.

In the following, simulation results are shown. Figure 10(a) shows the transition of objective function and Fig. 10 (b) shows the transitions of gas blow which are the control variables. In Fig. 10(c), estimated the temperature distribution is shown. The temperature difference of estimated one and desired one is shown in Fig. 10(d).

As shown here, the value of the objective function decreases with iterations. After 23 times iterations, it converges at 9.6. When the transition of gas flow is seen, the gas volume from tuyere \#4 becomes larger than others. Herewith, the estimated temperature distribution is high in the right of a center of a furnace. As for the difference of the estimated and the measured temperature, estimated one is equated to measured one at almost all nodes. The maximum difference is 7.2 degrees centigrade at the center of a

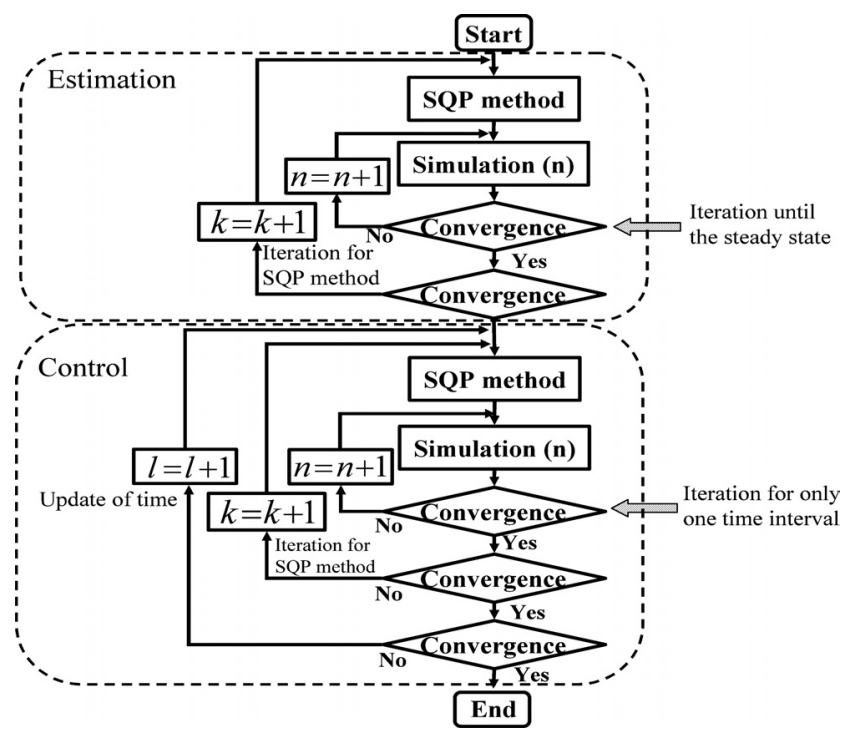

Fig. 9. Flow chart of estimation and control simulation.

Table 1. Measured data of instrumentations.

\begin{tabular}{|c||c|c|c|c|c|c|c|c|c|c|}
\hline & 1 & 2 & 3 & 4 & 5 & 6 & 7 & 8 & 9 & 10 \\
\hline$T\left(m_{t}\right)$ & 1189 & 1028 & 992 & 840 & 1391 & 1080 & 1003 & 845 & 1382 & 1383 \\
\hline$P\left(m_{p}\right)$ & 0.17 & 0.16 & 0.14 & 0.13 & 0.18 & 0.16 & 0.14 & 0.13 & - & - \\
\hline$V\left(m_{v}\right)$ & $(0.13,0.42)$ & $(-0.13,0.42)$ & - & - & - & - & - & - & - & - \\
\hline
\end{tabular}




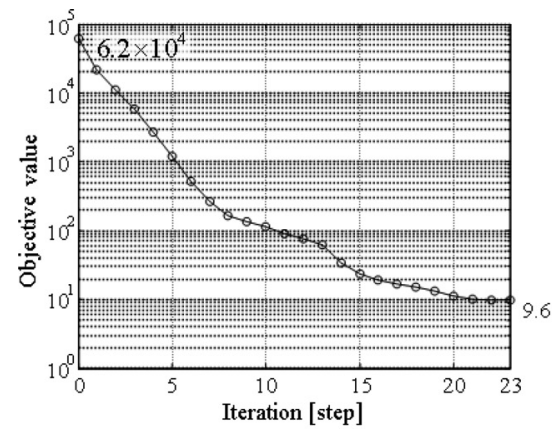

(a) Transition of objective function

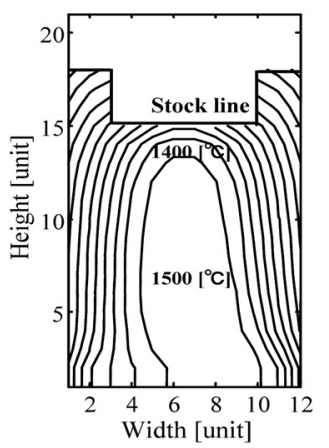

(c) Estimated temperature distribution

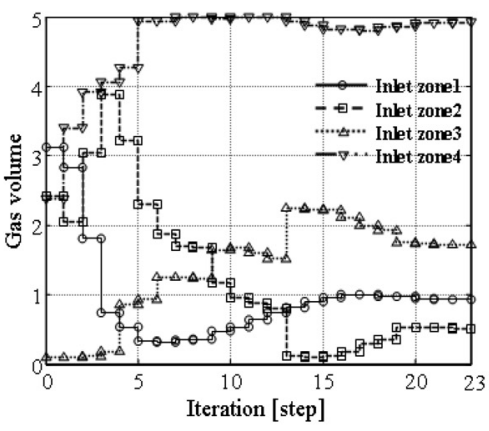

(b) Transition of decision variables

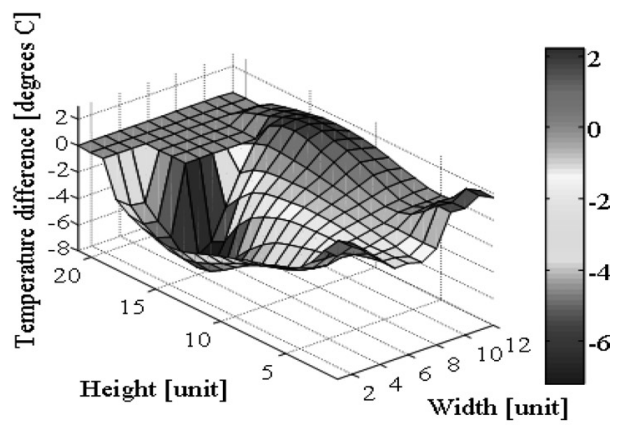

(d) Temperature difference

Fig. 10. Results of estimation.

reactor furnace. Temperature differences of the left side become larger than the right side. Near the position of instrumentations, the temperature difference is small and as it is pulled away from instrumentations, the temperature difference becomes large.

\subsection{Control of Temperature Distribution}

The control of temperature distribution in a reactor furnace is calculated by the method shown in Fig. 4. The initial temperature distribution and the desired temperature distribution are shown in Figs. 11 and $\mathbf{1 2}$ respectively.

The temperature distribution in a reactor furnace is controlled so as to attain the desired temperature distribution by changing supply values the operation of gas volumes from each tuyeres and materials from top of a furnace.

In the following, simulation results are shown. The transition of objective function, the transitions of gas blow which is control variables and the transitions of supplying material size which is control variables are shown in Figs. 13(a), 13(b) and 13(c) respectively. In Fig. 13(d), the optimized temperature distribution is shown. In Fig. 13(e), the temperature difference between the desired and the optimized temperature distribution is shown.

As for the transition of gas flow from tuyeres, gas volumes from tuyere \#1 and \#4 decrease slightly and them from tuyere \#2 and \#3 increase slightly in early time. Then, gas volumes from tuyere \#1 and \#4 increase slightly and them from tuyere \#2 and \#3 decrease slightly. When gas volume from tuyere \#1 and them from tuyere \#4 are compared, gas volume from tuyere \#4 become more than it from tuyere \#1. Finally, gas volumes of all tuyeres become about 2 units. When the transition of supplying material

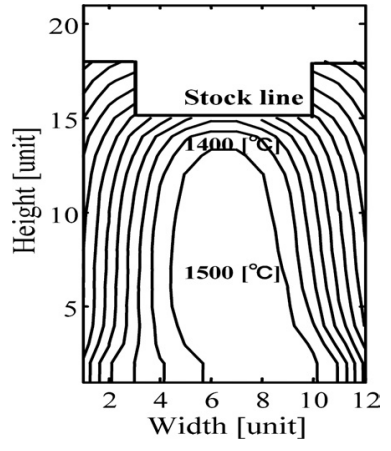

Fig. 11. Initial temperatures distribution.

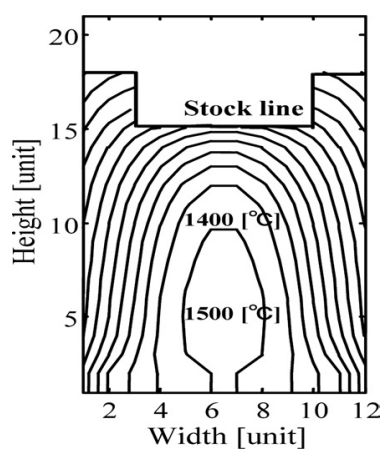

Fig. 12. Desired temperatures distribution.

size is seen, both sides of material size become large in early time. As time passes, the material size reduces from 0.067 meters to $0.047 \mathrm{~m}$ at both sides. The objective function decreases at every iterations. The temperature difference between the desired and the optimized temperature distribution is 6.5 degrees centigrade at the maximum. 


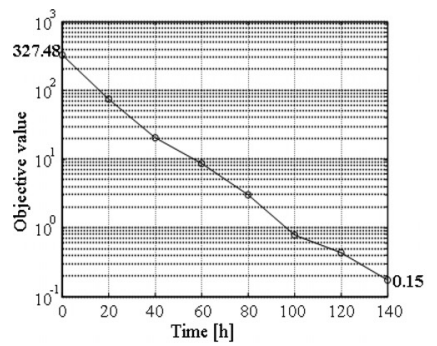

(a) Objective function

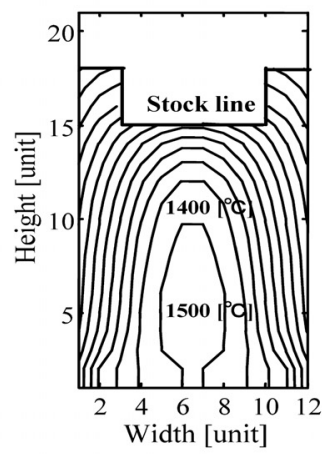

(d) Optimized temperature distribution

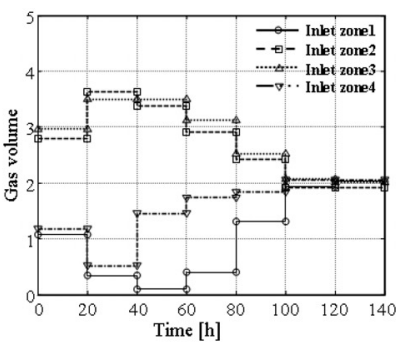

(b) Gas volume

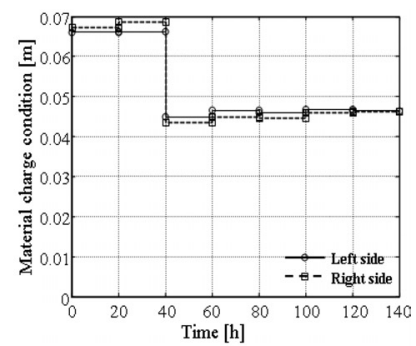

(c) Material size

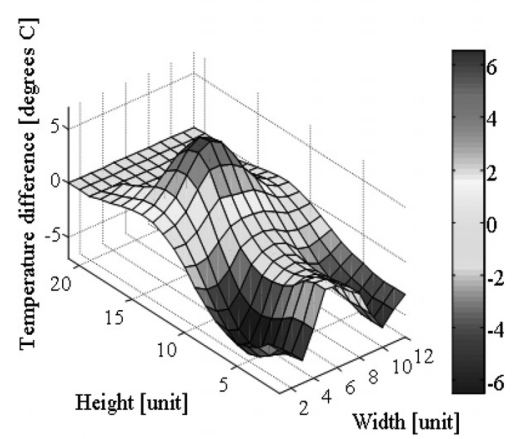

(e) Temperature difference

Fig. 13. Results of control.
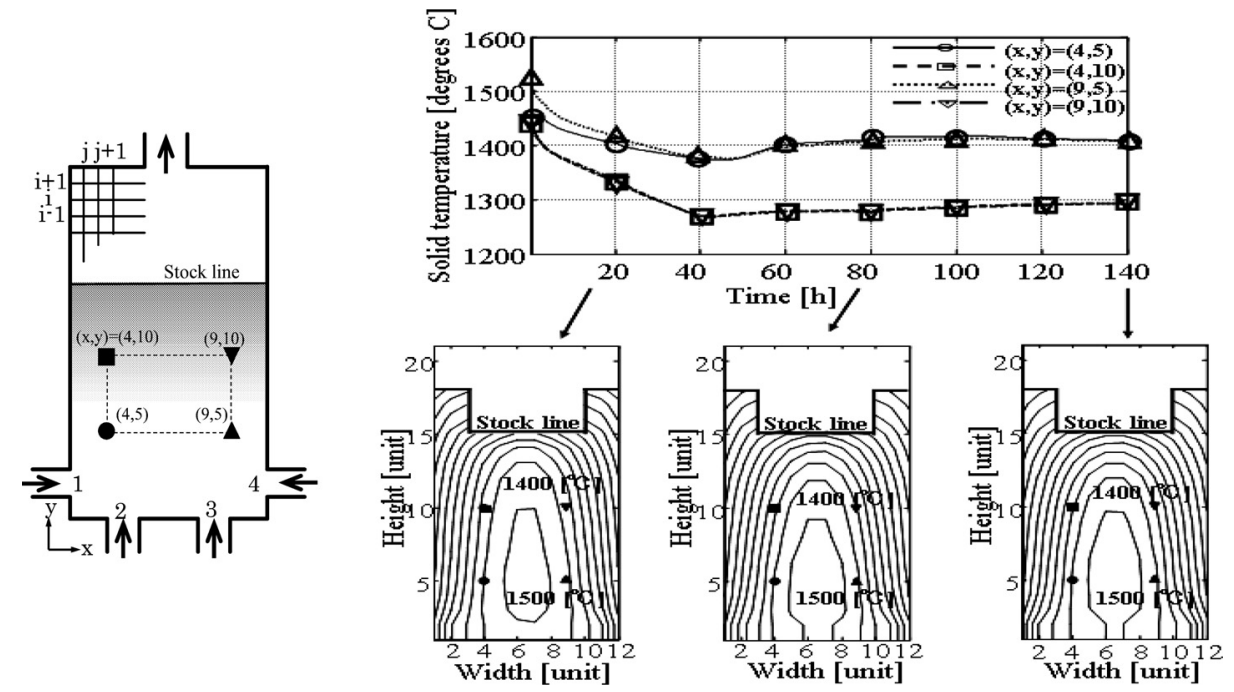

Fig. 14. Transition in temperatures at 4 nodes.

Transitions in furnace solid temperatures during control at four nodes are shown in Fig. 14. As shown in the figure, temperatures at these nodes are regulated to their desired values after $40 \mathrm{~h}$.

Furthermore, the result to have been controlled not temperatures of all nodes to be obtained by the estimated temperature distribution in a reactor furnace but measured temperatures are shown at the following. This objective function is described at Eq. (20). The change of Eq. (20) from Eq. (5) is the number of positions evaluated temperature.

$$
\begin{aligned}
f^{(l)}= & w_{1} \sum_{m_{\mathrm{t}}}\left(T_{\mathrm{d}}(i, j)-\hat{T}_{\mathrm{s}}^{(l)}(i, j)\right)^{2} \\
& +w_{2} \max \left\{\left|u_{h}^{(l+1)}-u_{h}^{(l)}\right|-a, 0\right\} .
\end{aligned}
$$

In the following, simulation results are shown.
Simulation conditions are the same as the above. The transition of objective function, the transitions of gas blow which is control variables and the transitions of supplying material size which is control variables are shown in Fig. 15(a), 15(b) and 15(c) respectively. In Fig. 15(d), the optimized temperature distribution is shown. In Fig. 15(e), the temperature difference between the desired and the optimized temperature distribution is shown.

As in Fig. 15(a) the calculation was not able to fulfill convergence conditions until 20 times iterations. Seeing from Figs. 11 and 12, Temperature in a reactor furnace is higher than the desired temperature in the early stages. As in Fig. 15(b), gas volume from tuyere \#1 and \#4 set at near instrumentation points is reduced so as to decrease furnace heat. As shown in Fig. 15(c), the supplying material size becomes large for the same purpose. After three iterations, 


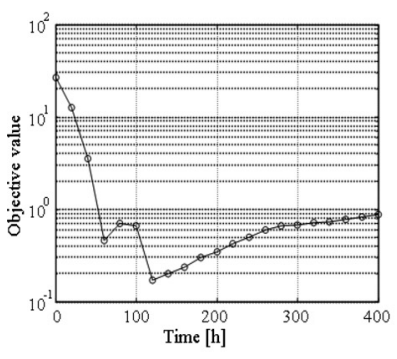

(a) Objective function

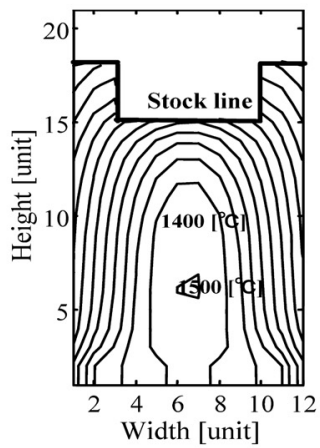

(d) Optimized temperature distribution

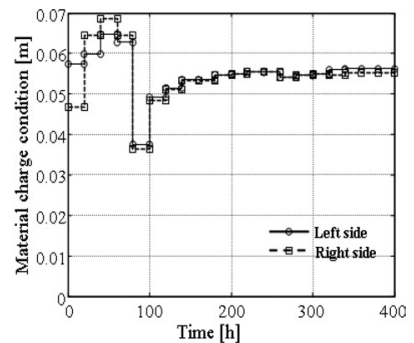

(c) Material size

(b) Gas volume

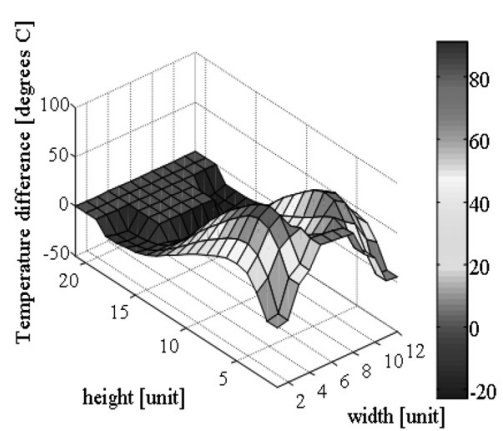

(e) Temperature difference

Fig. 15. Results of control without estimation.

the temperature difference decreases. However, because of the large time delay in heat transfer phenomenon, the inner temperature decreases too much. Then, gas volumes from tuyere \#1 and \#4 rise and supplying materials size becomes small in order to raise the furnace heat. In the process, gas volumes from tuyere \#2 and \#3 are decreasing and the temperature of the central part of a reactor furnace becomes smaller than the desired temperature. Therefore, as shown in Fig. 15(e), the value of temperature difference is the largest at the central part and is 91.4 degrees centigrade. Further, as shown in Fig. 15(d), the temperature in the central part of a furnace differs from its desired value.

As the results, it is verified that the estimation of temperature distribution is needed in order to control the temperature distribution in wide area of a reactor furnace.

\section{Conclusion}

In this paper, methods for estimating the inner furnace temperature distribution and for controlling gas blown from multiple tuyeres and supplying material to achieve the desired temperature distribution are proposed. For these purposes, the SQP method which is an effective method in solving nonlinear programming problem is used both to estimate the temperature distribution in a reactor furnace and to determine the control variables. Through numerical experiment, it has been confirmed that temperature distribution in a furnace can be estimated, even if there are some gaps in measured data. Furthermore, control variables such as gas volume and the supply of the material for achieving desired temperature distribution can be determined. Timeoptimal control of temperature distribution in a reactor furnace will be left for a future study.

\section{REFERENCES}

1) S. Ergun: Chem. Eng. Prog., 48 (1952), 89.

2) M. Kawakami, K. Murayama, M. Shibata, T. Takenaka and H. Toda: Tetsu-to-Hagané, 87 (2001), 45.

3) S. Watakabe and K. Takeda: Tetsu-to-Hagané, 87 (2001), 467.

4) A. Kasai, K. Miyagawa, T. Kamijo, J. Kiguchi and M. Shimizu: Tetsu-to-Hagané, 83 (1997), 239.

5) M. Konishi, S. Tamura, Y. Otsuka, R. Hori and R. Ono: Kobe Steel Eng. Rep., 37 (1987), 3.

6) K. Takatani, Y. Ujisawa and T. Inada: Modeling and Control of Large-scale Complex Process with Reactions, ISIJ, Tokyo, (1996), 27.

7) K. Tsumura: Modeling and Control of Large-scale Complex Process with Reactions, ISIJ, Tokyo, (1996), 33.

8) T. Shibuta, M. Konishi, J. Imai and T. Nishi: CAMP-ISIJ, 15 (2002), 925.

9) K. Ishimaru, M. Konishi, J. Imai and T. Nishi: CAMP-ISIJ, 17 (2004), 174.

10) K. Ishimaru, M. Konishi, J. Imai and T. Nishi: Proc. of 46th JACC, ISCIE, Kyoto, (2003), 54.

\section{Appendix. Mathematical Models}

The following is described about the mathematical equation applied to the furnace model.

\section{Gas Flow Model}

In gas flow, heat is conveyed with movement of fluid. In other words, when fluid flow is rapid, a lot of heat is conveyed along the direction. Since the same condition holds for the inside of reactor furnace, gas flow from tuyeres reveals much effect on the dispersion of heat. Here, the mathematical model to find the gas flow distribution is explained.

Ergun equation accompanying flow stress is adopted as the equation to find the gas flow distribution. This is described in Eq. (A-1). 


$$
\frac{\partial V}{\partial t}=-2 \nabla p-\left(f_{1}+f_{2}|V|\right) V+\frac{1}{\operatorname{Re}} \Delta V
$$

Where, $V$ is velocity of gas flow, $p$ is pressure, $\mathrm{Re}$ is Reynolds number, $f_{1}$ and $f_{2}$ are coefficients of Ergun equation.

$$
f_{1}=150 \frac{(1-\varepsilon)^{2}}{\varepsilon^{3}} \frac{\mu}{D_{\mathrm{p}}^{2}}, \quad f_{2}=1.75 \frac{1-\varepsilon}{\varepsilon^{3}} \frac{\mu}{D_{\mathrm{p}}} \ldots
$$

$\varepsilon$ : Fractional void volume, $\rho$ : Density of gas, $\mu$ : Absolute viscosity of gas, $D_{\mathrm{p}}$ : Diameter of material particle

\section{Temperature Model}

We think of gas and solid layer to find the results of inner temperature distribution in reactor furnace. Equation to find the gas temperature distribution in reactor furnace is described by Eq. (A-3).

$$
\frac{\partial T_{\mathrm{g}}}{\partial t}=-(V \cdot \nabla) T_{\mathrm{g}}+\lambda_{\mathrm{g}} \Delta T_{\mathrm{g}}-h\left(T_{\mathrm{g}}-T_{\mathrm{s}}\right) \ldots \ldots \ldots(\mathrm{A}-3
$$

Where, $T_{\mathrm{g}}$ is gas temperature, $T_{\mathrm{s}}$ is solid temperature, $\lambda_{\mathrm{g}}$ is gas thermal conductivity and $h$ is heat transfer coefficient between gas and solid. Equation (A-3) is made or described based on the relation for heat conduction adding heat advection in the first term of right-hand side and the heat transfer between solid and gas in the third term of righthand side respectively.

Equation for the solid temperature distribution in reactor furnace is described by Eq. (A-4).

$$
\frac{\partial T_{\mathrm{s}}}{\partial t}=\lambda_{\mathrm{s}} \Delta T_{\mathrm{s}}+k \cdot Q+h\left(T_{\mathrm{g}}-T_{\mathrm{s}}\right)
$$

Where, $\lambda_{\mathrm{s}}$ is solid thermal conductivity, $Q$ is heat of raw material by reaction and $k$ is reactivity coefficient. Equation (A-4) is made based on equation of heat conduction, too. Moreover, Eq. (A-4) is additively made the heat of raw material in the second term of right-hand side and the heat transfer between solid and gas in the third term of righthand side respectively.

\section{Parameters for Equations}

Generally, reaction of raw material starts with high temperature therefore reactivity coefficient $k$ is enlarged in high temperature. However, iron ores in raw material are dissolved at about 1300 to 1400 degrees $C$ and it is reduced to pig-iron. This reactivity coefficient $k$ is defined by Eq. (A5). It is associated with material size and temperature in a reactor furnace.

$$
\begin{aligned}
& k=\frac{\alpha}{D_{\mathrm{p}}} \cdot \exp \left(-\frac{E}{R \cdot T_{\mathrm{g}}}\right) \cdot \beta V k^{\prime} \\
& k^{\prime}=\frac{1}{1+\exp \left\{\left(T_{\mathrm{g}}-1700\right) / \gamma\right\}}
\end{aligned}
$$

$R$ : Gas constant, $E$ : Activity energy, $\alpha, \beta, \gamma$ : Constant 